\title{
Is Hypothermia Helpful in Severe Subarachnoid Hemorrhage? An Exploratory Study on Macro Vascular Spasm, Delayed Cerebral Infarction and Functional Outcome after Prolonged Hypothermia
}

\author{
Joji B. Kuramatsu ${ }^{a}$ Rainer Kollmar ${ }^{a}$ Stefan T. Gerner ${ }^{a}$ Dominik Madžara \\ Andrea Pisarčíková ${ }^{a}$ Dimitre Staykov ${ }^{a}$ Stephan P. Kloskab Arnd Doerfler ${ }^{b}$ \\ Ilker Y. Eyüpogluc Stefan Schwaba Hagen B. Huttner ${ }^{a}$ \\ Departments of a Neurology, ${ }^{b}$ Neuroradiology and ${ }^{\mathrm{C}}$ Neurosurgery, University of Erlangen-Nuremberg, Erlangen, Germany
}

\author{
Key Words \\ Subarachnoid hemorrhage · Vasospasm - Delayed cerebral \\ infarction $\cdot$ Hypothermia
}

\begin{abstract}
Background: Therapeutic hypothermia (TH) is an established treatment after cardiac arrest and growing evidence supports its use as neuroprotective treatment in stroke. Only few and heterogeneous studies exist on the effect of hypothermia in subarachnoid hemorrhage $(\mathrm{SAH})$. A novel approach of early and prolonged $\mathrm{TH}$ and its influence on key complications in poor-grade $\mathrm{SAH}$, vasospasm and delayed cerebral ischemia (DCl) was evaluated. Methods: This observational matched controlled study included 36 poor-grade (Hunt and Hess Scale $>3$ and World Federation of Neurosurgical Societies Scale $>3$ ) SAH patients. Twelve patients received early $\mathrm{TH}\left(<48 \mathrm{~h}\right.$ after ictus), mild $\left(35^{\circ} \mathrm{C}\right)$, prolonged (7 \pm 1 days) and were matched to 24 patients from the prospective SAH database. Vasospasm was diagnosed by angiography, macrovascular spasm serially evaluated by Doppler sonography and $\mathrm{DCl}$ was defined as new infarction on follow-up CT. Functional outcome was assessed at 6 months
\end{abstract}

by modified Rankin Scale (mRS) and categorized as favorable (mRS score $0-2$ ) versus unfavorable (mRS score 3-6) outcome. Results: Angiographic vasospasm was present in $71.0 \%$ of patients. TH neither influenced occurrence nor duration, but the degree of macrovascular spasm as well as peak spastic velocities were significantly reduced $(p<0.05)$. Frequency of DCl was $87.5 \%$ in non-TH vs. $50 \%$ in $\mathrm{TH}$-treated patients, translating into a relative risk reduction of $43 \%$ and preventive risk ratio of $0.33(95 \% \mathrm{Cl} 0.14-0.77, \mathrm{p}=0.036)$. Favorable functional outcome was twice as frequent in $\mathrm{TH}$ treated patients 66.7 vs. $33.3 \%$ of non-TH $(p=0.06)$. Conclusion: Early and prolonged TH was associated with a reduced degree of macrovascular spasm and significantly decreased occurrence of $\mathrm{DCl}$, possibly ameliorating functional outcome. TH may represent a promising neuroprotective therapy possibly targeting multiple pathways of DCl development, notably macrovascular spasm, which strongly warrants further evaluation of its clinical impact.

(c) 2015 S. Karger AG, Basel

J.B.K. and R.K. contributed equally to this investigation.

\section{KARGER 125}

(c) 2015 S. Karger AG, Base

$1015-9770 / 15 / 0406-0228 \$ 39.50 / 0$

E-Mail karger@karger.com

www.karger.com/ced
Dr. Joji B. Kuramatsu

Department of Neurology

University of Erlangen, Schwabachanlage 6

DE-91054 Erlangen (Germany)

E-Mail Joji.Kuramatsu@uk-erlangen.de 


\section{Introduction}

Occurrence of delayed cerebral ischemia (DCI) in subarachnoid hemorrhage (SAH) is a major contributor to morbidity and mortality $[1,2]$. Recent research increased understanding of mechanisms underlying DCI reflecting a multifactorial process, that is, inflammation, microthrombosis, disturbed autoregulation, spreading depolarization and cerebral vasospasm [2-5]. Yet, within the last few decades, randomized controlled trials (RCTs) investigating treatment strategies for DCI have failed to improve clinical end points [1]. Thus, development of a more comprehensive treatment approach targeting multiple pathways seems necessary $[2,5]$.

Therapeutic hypothermia (TH) has been shown to modulate various pathways including molecular, metabolic and inflammatory processes [6]. TH has been questioned recently but still remains an accepted neuroprotective treatment after cardiac arrest [7, 8]. An currently ongoing RCT evaluates TH as adjunctive therapy to systemic thrombolysis in ischemic stroke (http://www. eurohyp1.org) [11] based on neuroprotective effects described in both ischemic and hemorrhagic stroke patients $[6,9,10]$. Only few experimental and clinical reports exist for patients with $\mathrm{SAH}$ treated with $\mathrm{TH}$, mainly describing a reduction of intracranial pressure (ICP) [12-14]. However, interpretation remains limited as varying treatment modalities, heterogeneous protocols and patient populations were investigated.

Detailed analyses of $\mathrm{TH}$-induced effects after SAH are lacking. The present study was designed to investigate a novel approach of early and prolonged $\mathrm{TH}$ on the key complications - vasospasm and DCI - in poor-grade SAH.

\section{Methods}

\section{Patient Selection and Study Design}

All patients admitted to the neurocritical care unit during the years 2010-2012 were screened for eligibility. Inclusion criteria for this exploratory pilot study consisted of: aneurysmal SAH with complete aneurysm closure within $24 \mathrm{~h}$, poor-grade SAH defined as Hunt and Hess-Scale 4-5, World Federation of Neurosurgical Societies Scale 4-5 and modified Fisher Scale 3-4, necessity for external ventricular drainage because of hydrocephalus, obligatory sedation and mechanical ventilation, absence of brainstem symptoms, initiation of $\mathrm{TH}$ with achievement of target temperature within $48 \mathrm{~h}$ after symptom onset. Exclusion criteria consisted of: clinical instability (i.e. new-onset brainstem symptoms, referring to disturbed brainstem reflexes, that is, pupillary light reflex, corneal reflex, pharyngeal reflex) within the first $24 \mathrm{~h}$ upon neurocritical care unit admission, exceeding the pre-defined time window for initiation of $\mathrm{TH}$ (e.g. longer transfer from peripheral hospitals, etc.) and incompletely obliterated aneurysm or evidence of additional, non-ruptured, non-treated aneurysms. The local ethics committee of the University Hospital ErlangenNuremberg, Germany, approved the study. Informed consent was obtained from relatives or legal representatives to receive $\mathrm{TH}$ as individual 'rescue therapy' for the participating critically ill SAH patients.

A matched controlled design was used to compare (i) characteristics of cerebral macrovascular spasm, (ii) occurrence of DCI during hospital stay, (iii) mortality and (iv) functional outcome. Matching procedure applied 1:2 ratio matching according to following prioritization process: amount of SAH and intraventricular hemorrhage (modified Fisher Scale and Hijdra score), clinical status on admission (GCS, Hunt and Hess, World Federation of Neurosurgical Societies) and patient age. Hence, 12 patients received $\mathrm{TH}$ and were matched to 24 patients from a prospective institutional SAH database (patients admitted during 2010-2012 fulfilling above-mentioned inclusion and exclusion criteria). We obtained all clinical parameters through our prospective institutional database. Functional outcome was assessed by the modified Rankin Scale (mRS) 6 months after disease onset. For outcome evaluation, we conducted a semi-quantitative telephonic interview directly with the patients, their legal representatives or their closest relatives. A favorable outcome was defined as mRS score of 0-2 and unfavorable outcome as mRS score of 3-6.

\section{TH Protocol}

The pre-specified TH protocol employed: TH induction within $48 \mathrm{~h}$ after symptom onset, to a target body core temperature of $35^{\circ}$ (measured by bladder catheter), for a prolonged period of $7 \pm$ 1 days. This protocol targeted the multiple pathophysiological mechanisms of DCI [2], very early in development-phase, for a prolonged period based upon reasonable safety profile of mild TH [10] and to cover the known time period of peak vasospasm generation. Endovascular cooling was carried out by central line catheter $\left(\right.$ ICY $^{\circledR}$, IC-3893, Zoll-Medical) that was placed in the femoral vein according established protocols [10]. Shivering during TH was treated at Bedside Shivering Assessment Scale score $>1$ initially with deeper sedation or consecutively with pethidine infusions and muscle relaxants in refractory cases [15]. We carried out a step-wise re-warming process with a maximal temperature increase of $0.5^{\circ} \mathrm{C}$ over $24 \mathrm{~h}$. Screening for $\mathrm{TH}$-associated complications, coagulopathy and thrombosis, infections (pneumonia, sepsis), enzyme elevation ( $\alpha$-amylase, AST, ALT), electrolyte disorders and cardiac arrhythmias was performed on a daily basis [16].

\section{General Neurocritical Care Unit Treatment}

Patients received standard medical therapy according to guidelines $[1,17]$. Aneurysms were occluded by endovascular approach or neurosurgical clipping within the first $24 \mathrm{~h}$ of admission. We treated ICP levels greater $20 \mathrm{~mm} \mathrm{Hg}$ with hypertonic saline or mannitol infusions monitored by external ventricular drainage or parenchymal probes [18]. All patients were sedated using midazolam or propofol in combination with sufentanil for analgesia. General temperature management for the control group as well as for the TH group after re-warming consisted of paracetamol $(1 \mathrm{~g})$ infusions if body core temperature raised over $38.3^{\circ} \mathrm{C}$. Intravenous nimodipine was continuously administered 
at a dose of $2 \mathrm{mg} / \mathrm{h}$ immediately after admission and was switched to oral application as soon as possible. A cerebral perfusion pressure of $70 \mathrm{~mm} \mathrm{Hg}$ was targeted maintaining euvolemia and controlling blood pressure using catecholamine support, if necessary. Hemodilution was not applied and packed RBC transfusions were given at restrictive thresholds $(\mathrm{Hb}<8 \mathrm{~g} / \mathrm{dl})$. Lung protective mechanical ventilation targeted $\mathrm{PaCO}_{2}$ levels between $35-40 \mathrm{~mm} \mathrm{Hg}$ as measured according to the $\mathrm{pH}$-Stat methodology [19].

\section{Imaging}

Initial diagnosis of SAH was based on CT (SOMATOM Definition AS+, Siemens, Erlangen, Germany) and aneurysms identified using diagnostic subtraction angiography. Two neuroradiologists blinded to clinical data, with access to information such as date, time and methodology of endovascular procedures and surgical treatment, evaluated the scans independently and scored neuroradiological parameters $[20,21]$. In cases of discrepant scoring, a second consensus analysis was carried out. Initial follow-up imaging was performed within $24 \mathrm{~h}$ and consecutive scans were carried out at $48-72 \mathrm{~h}$ intervals.

\section{Doppler Investigations, Macrovascular Spasm and DCI}

Three certified physicians performed routine extracranial and transcranial Doppler examinations using $2-\mathrm{MHz}$ (pulsedwave) and 4-MHz (continuous-wave) probes (Sonara/tek, MediLab, Würzburg, Germany) starting on the day of admission and routinely every $24-48 \mathrm{~h}$ thereafter up to a maximum of 21 days (a total of $n=337$ Doppler examinations). All cerebral vessels were insonated at specific depths for the assessment of flow velocities (e.g. peak flow velocities) and were converted by validated conversion formula, as previously described [22]. We used middle cerebral artery (MCA) velocities to compare possible treatment effects between patients receiving $\mathrm{TH}$ and those not receiving TH [23]. Definition of Doppler-based vasospasm, referred to as macrovascular spasm throughout the manuscript consisted of present mean flow velocities greater than $120 \mathrm{~cm} / \mathrm{s}$ and/or a Lindegaard ratio higher than 3 , which was chosen for early detection [1, 17, 22, 24]. Macrovascular spasm was confirmed as angiographic vasospasm by diagnostic subtraction angiography $(\mathrm{n}=15), \mathrm{CT}$ angiography and perfusion scanning $(\mathrm{n}=$ $25)$ as well as by MRI $(n=6)$. The definition of DCI was exclusively based on native follow-up CT imaging and required a newly demarcated cerebral infarction. Attentive care was taken to differentiate DCI from peri-procedural lesions associated to interventional procedures or microsurgical repair. A new onset of clinically relevant focal neurological deficit was not assessable, since all patients' required mechanical ventilation during stay. For DCI detection, a median number of 8 (95\% CI 3-12) CT scans was available.

\section{Statistical Analysis}

Statistical Analysis was performed with SPSS 20.0 (www.spss. com). The significance level was set at $\alpha=0.05$. Corrections for multiple comparisons were not applied in relation to the exploratory nature of this investigation. Statistical tests were 2-sided. The Kolmogorov-Smirnov test was applied to determine distribution of the data. Latter are presented as mean \pm SD (compared using the Student $t$ test) or as median and interquartile range (compared using the Mann-Whitney $U$ test), as appropriate. The Pearson $\chi^{2}$ and the Fisher's exact tests were used to compare frequency distributions of categorized variables between patients treated with $\mathrm{TH}$ as opposed to those without TH. Corresponding ORs with 95\% CIs are given for the analysis of investigated end points, that is, DCI and functional outcome.

\section{Results}

Table 1 compares matching parameters, baseline characteristics and risk factors for DCI development [25]. No significant differences were noted between both groups. Especially, in TH-treated patients, the rate of thromboembolic complications was not different. In TH patients, statistical trends were documented for a shorter duration of hospital stay (TH: $21.8( \pm 9.2)$ vs. Ø-TH: $32.4( \pm 23.5)$; $\mathrm{p}=0.090)$ and lower rate of ventriculoperitoneal shunt surgery (TH: 1 (8.3\%) vs. Ø-TH: 8 (40.0\%); p =0.059). Sub-analyses showed that ventriculoperitoneal shunt dependency was most strongly associated with length of hospital stay (receiver operating characteristic analysis: $\mathrm{AUC}=0.924,95 \%$ CI 0.840-1.00, $\mathrm{p}<0.001$ ) likely mediating the observed difference of longer hospital stay in untreated patients. In treated patients, target temperature was reached within $48 \mathrm{~h}$ and remained stable during the treatment phase (fig. 1). Body temperature remained significantly reduced beyond the period of intravascular cooling with differences up to day 18 in treated patients $(\mathrm{p}<0.05)$.

\section{Characteristics of Cerebral Macrovascular Spasm}

For the investigated cohort of 36 patients, a total of 337 Doppler examinations were performed.

Frequency of Macrovascular Spasm. Doppler-based macrovascular spasm was present in $83.3 \%$ of TH patients vs. $87.5 \%$ in non-TH patients. Verification by other diagnostic modalities resulted in a vasospasm rate for TH-treated patients of $60.0 \%$ vs. untreated patients $76.2 \%$ ( $\mathrm{p}=0.302$; table 2$)$.

Onset and Duration of Macrovascular Spasm. Figure 2 displays the comparison of MCA velocities providing an overview of time course and degree of Doppler-based macrovascular spasm. Macrovascular spasm occurred at 3-4 days in all patients, and the duration of macrovascular spasm in TH was $13.3 \pm 5.4$ days vs. $16.1 \pm 3.5$ in nonTH patients $(\mathrm{p}=0.108)$.

Degree of Macrovascular Spasm. A significant reduction of mean MCA velocities was noted on both insonated hemispheres in TH-treated patients $(\mathrm{p}<0.05)$. This difference was apparent between days 7-12 (fig. 2). 
Table 1. Matching characteristics, DCI risk factors and in-hospital measures for all analyzed TH vs. non-TH SAH patients

\begin{tabular}{|c|c|c|c|}
\hline SAH $(n=36)$ & $\mathrm{TH}(\mathrm{n}=12)$ & $\emptyset-\mathrm{TH}(\mathrm{n}=24)$ & $\mathrm{p}$ value \\
\hline \multicolumn{4}{|l|}{ Matching characteristics } \\
\hline Age, years ${ }^{\dagger}$ & $48.9 \pm 9.6$ & $51.3 \pm 11.9$ & 0.289 \\
\hline Glasgow coma scale & $8(3-14)$ & $8(4-13)$ & 0.779 \\
\hline Hunt and hess-scale f & $4(4-5)$ & $4(4-5)$ & 0.856 \\
\hline World federation of neurosurgical societies-scale & $4(4-5)$ & $4(4-5)$ & 0.679 \\
\hline Modified-fisher-scale ${ }^{\ddagger}$ & $4(3-4)$ & $4(3-4)$ & 0.212 \\
\hline \multicolumn{4}{|l|}{ Neuroradiological data } \\
\hline Anterior communicating artery aneurysm* & $6(50.0)$ & $11(45.8)$ & 0.806 \\
\hline MCA aneurysm* & $3(25.0)$ & $5(20.8)$ & 0.544 \\
\hline Posterior circulation aneurysm* & $3(25.0)$ & $8(33.3)$ & 0.456 \\
\hline Coiling of aneurysm* & $10(83.3)$ & $21(87.5)$ & 0.549 \\
\hline \multicolumn{4}{|l|}{ Risk factors for DCI } \\
\hline Hyperglycemia* & $7(58.3)$ & $13(54.2)$ & 0.806 \\
\hline Acute hydrocephalus* & $10(83.3)$ & $18(75.0)$ & 0.691 \\
\hline History of hypertension* & $8(66.7)$ & $13(54.2)$ & 0.475 \\
\hline Hemoglobin, $\mathrm{mmol} / \mathrm{l}^{\dagger}$ & $8.4 \pm 0.8$ & $7.8 \pm 1.4$ & 0.246 \\
\hline \multicolumn{4}{|l|}{ In-hospital measures and complications } \\
\hline Gender*, o & $9(75.0)$ & $15(62.5)$ & 0.359 \\
\hline Duration of ventilation $^{\dagger}$, days & $20.1 \pm 10.3$ & $18.8 \pm 15.3$ & 0.760 \\
\hline Tracheostomy* & $8(66.7)$ & $8(33.3)$ & 0.058 \\
\hline Pneumonia* & $10(83.3)$ & $15(62.5)$ & 0.268 \\
\hline Ventriculitis* & $2(16.7)$ & $3(20.8)$ & 1 \\
\hline Duration of $\mathrm{EVD}^{\dagger}$, days & $15.5 \pm 7.4$ & $19.5 \pm 10.7$ & 0.312 \\
\hline Osmotherapy* & $11(91.7)$ & $21(87.5)$ & 0.592 \\
\hline Length of stay ${ }^{\dagger}$, days & $21.8 \pm 9.2$ & $32.4 \pm 23.5$ & 0.090 \\
\hline In-hospital mortality* & $2(16.7)$ & $4(16.7)$ & 1 \\
\hline
\end{tabular}

${ }^{*} \mathrm{n}(\%) ;{ }^{\dagger}$ mean $\pm \mathrm{SD} ;{ }^{\ddagger}$ median (IQR $25-75$ th percentile).

\section{Occurrence of DCI}

As shown in table 2, a significantly reduced rate of DCI was observed in TH-treated patients (TH: 50\% (n = 6 of 12$)$ vs. $\varnothing$-TH: $87.5 \%(\mathrm{n}=21$ of 24$) ; \mathrm{p}=0.036)$. This translated into a significant relative risk reduction of $43 \%$ and preventive risk ratio of 0.33 (95\% CI 0.14-0.77, $\mathrm{p}=0.036)$ for the development of DCI. No statistical differences were however seen for new demarcated lesions (median $2(1-4)$ per patient) nor its duration until occurrence between the 2 compared groups $(\mathrm{p}=0.604$, $\mathrm{p}=0.942$ ). DCI developed predominantly within the vascular territory of peak spastic vessels in all patients at a rate of $85.2 \%(n=23$ of 27$)$. Figure 3 displays the chronology of DCI occurrence and compares the influence of TH treatment on mean flow velocities in peak spastic vessels. Early TH significantly reduced peak flow velocities starting at day 5 lasting intermittently until day 20 ( $\mathrm{p}<0.05$; fig. 3 ). 


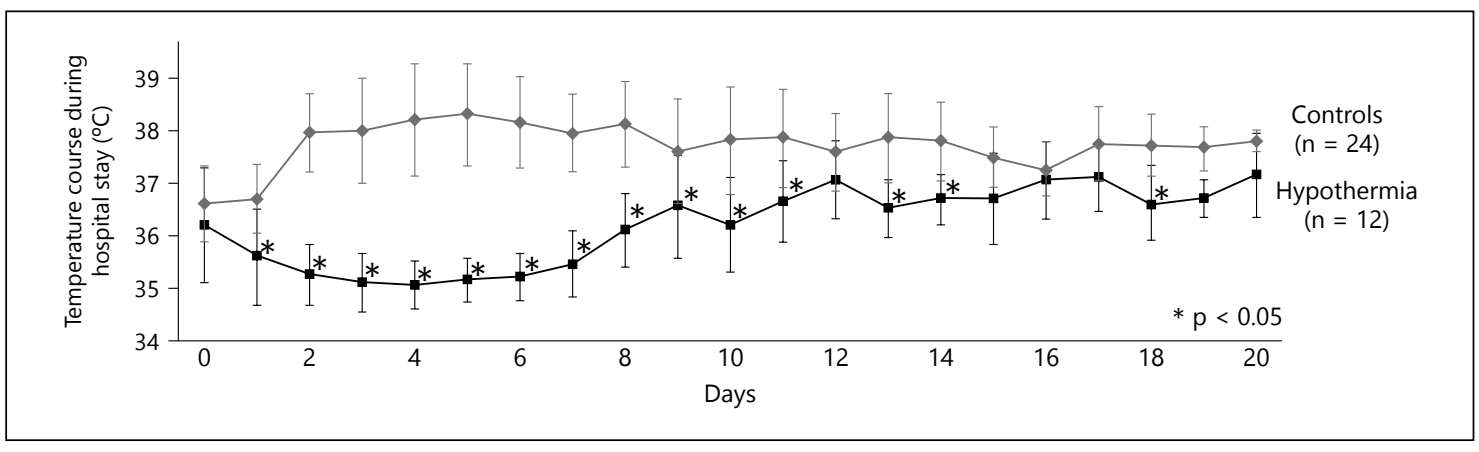

Fig. 1. Comparison of temperature course in TH vs. non-TH patients. Significantly different mean $( \pm S D)$ body core temperature values are marked by asterixis.

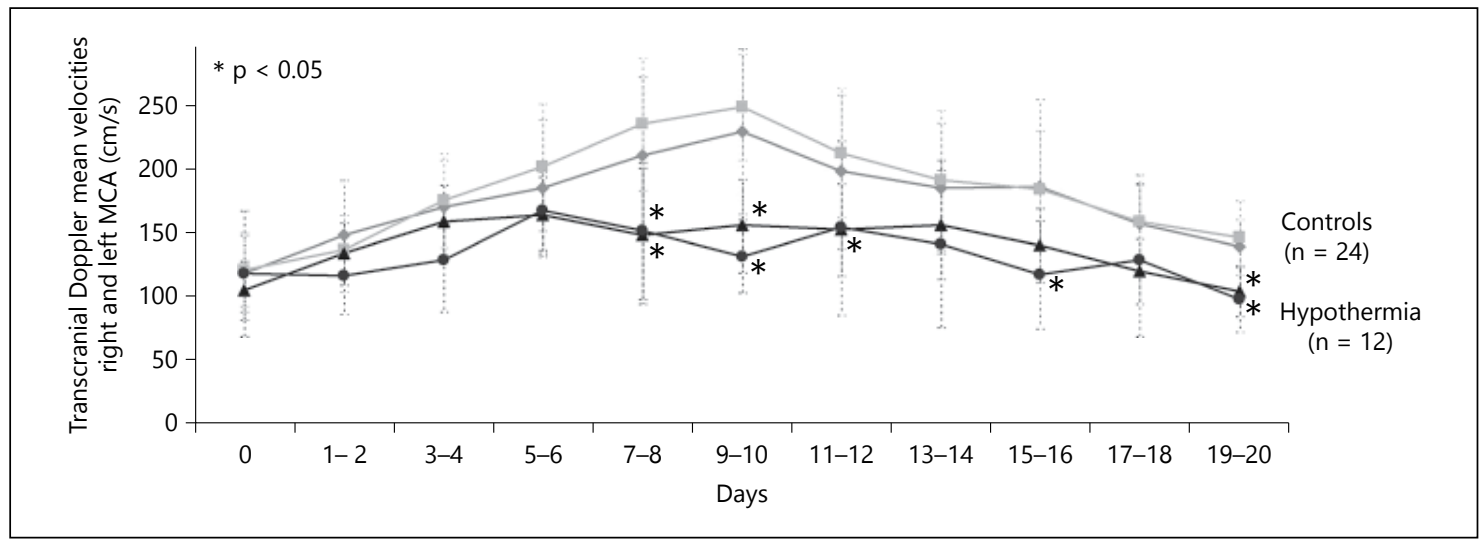

Fig. 2. Comparison of left and right MCA mean Doppler velocities in TH vs. non-TH patients. Significant differences in MCA mean $( \pm \mathrm{SD})$ velocities are marked by asterixis.

Table 2. Frequency of macrovascular spasm and characteristics of DCI in TH vs. non-TH patients

\begin{tabular}{|c|c|c|c|}
\hline SAH $(n=36)$ & $\mathrm{TH}(\mathrm{n}=12)$ & $\varnothing-\mathrm{TH}(\mathrm{n}=24)$ & $\mathrm{p}$ value \\
\hline Macrovascular spasm on TCD* & $10(83.3)$ & $21(87.5)$ & 1 \\
\hline Duration of macrovascular spasm ${ }^{\dagger}$, days & $13.3 \pm 5.4$ & $16.1 \pm 3.5$ & 0.108 \\
\hline Vasospasm on angiography* & $6 / 10(60.0)$ & $16 / 21(76.2)$ & 0.302 \\
\hline Delayed cerebral infarction* & $6(50.0)$ & $21(87.5)$ & 0.036 \\
\hline Total infarctions per patient with $\mathrm{DCI}^{\ddagger}$ & $2.2(1-4)$ & $1.9(1-3)$ & 0.604 \\
\hline Mean duration until $\mathrm{DCI}^{\dagger}$, days & $7.8 \pm 3.8$ & $8.0 \pm 4.2$ & 0.942 \\
\hline Infarction over peak spastic vessel* & $5 / 6(83.3)$ & $18 / 21(85.7)$ & 0.659 \\
\hline
\end{tabular}

Significant parameters are expressed in bold. ${ }^{*} \mathrm{n}(\%) ;{ }^{\dagger}$ mean $\pm \mathrm{SD} ;{ }^{\ddagger}$ median (IQR $25-75$ th percentile).

\section{Mortality and Functional Outcome}

No differences were seen for in-hospital mortality (TH: $16.7 \%(\mathrm{n}=2$ of 12$)$ vs. $\varnothing-\mathrm{TH}: 16.7 \%(\mathrm{n}=4$ of 24$)$; $\mathrm{p}=1$ ) but, as shown in figure 4 , the rate of favorable functional outcome ( $\mathrm{mRS}$ score $0-2$ ) at 6 months was doubled in TH-treated patients (TH: $66.7 \%(\mathrm{n}=8$ of 12$)$ vs. $\varnothing$-TH: $33.3 \%(\mathrm{n}=8$ of 24$) ; \mathrm{p}=0.06)$.

\section{Discussion}

The present investigation on early and prolonged $\mathrm{TH}$ in poor-grade SAH patients shows a reduced degree of macrovascular spasm, fewer occurrence of DCI and a possible association with improved functional outcome in treated patients. Two questions emerge from the data: 


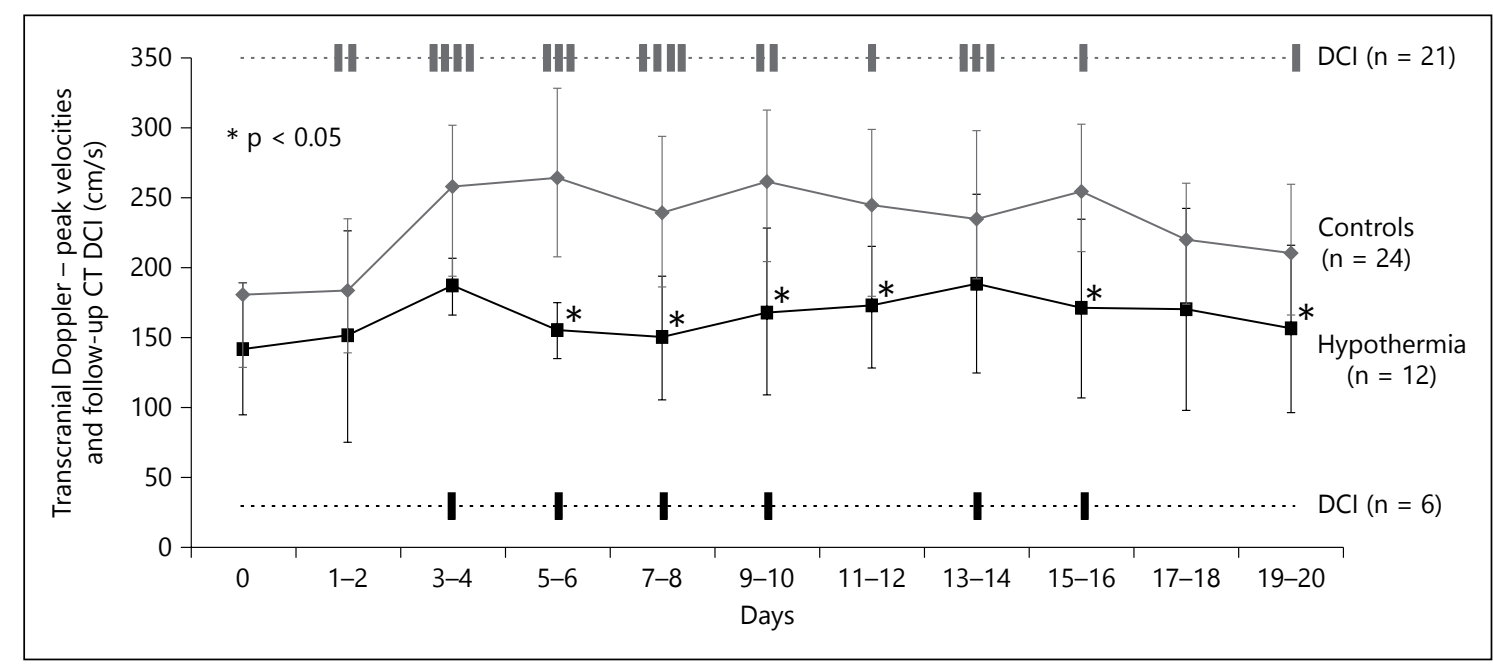

Fig. 3. Comparison of Doppler peak velocities (mean \pm SD) of all insonated vessels and chronological distribution of DCI over 21 days.

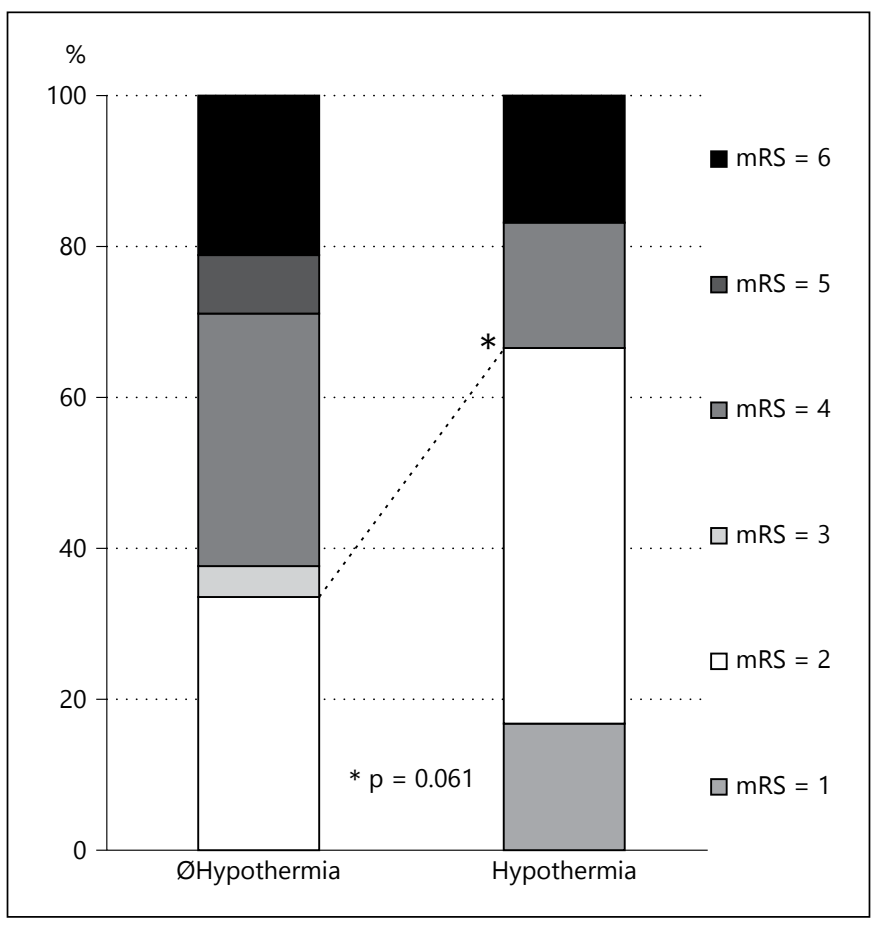

Fig. 4. Distribution of functional outcome as assessed by the mRS at 6 months comparing TH vs. non-TH patients.

how may $\mathrm{TH}$ modulate the degree of macrovascular spasm? and why is the occurrence of DCI reduced?

Angiographic vasospasm occur in up to $70 \%$ of patients after $\mathrm{SAH}$, yet less than half develop ischemic neurologic symptoms [2]. RCTs within the last decades investigating vasospasm treatment have failed to prevent DCI or to im-

Hypothermia in Poor-Grade SAH prove outcome $[26,27]$ questioning the causal relationship of vasospasm as sole contributor to DCI development [24]. The present data show that DCI occurred predominantly in the vascular territory of peak spastic vessels supporting the role of vasospasm. TH was related to significant reductions of MCA and peak spastic velocities, which may resemble a decreased degree of macrovascular spasm [23]. As previously suggested, TH mediates its neuroprotective effects by modulations of molecular, metabolic and inflammatory processes, possibly supporting associations with cerebral blood flow velocities [6, 12, 28-30]. Such a mechanistic association of TH seems likely, yet its influence on actual peak spasm and its clinical significance warrant further investigations [31]. Available clinical studies have investigated $\mathrm{TH}$ rather as acute treatment strategy for increased ICP than for its effects on vasospasm and DCI $[12-14,29,31]$. To date, no study has evaluated this novel approach of early and prolonged $\mathrm{TH}$ and its influence on those complications after poor-grade SAH.

Results here provide associations of early $\mathrm{TH}$ with a decreased risk and reduced rate of DCI. Possibly, this beneficial association may have translated into a doubled rate of favorable functional outcome in treated patients. All patients who exhibited DCI showed a median of 2 newly demarcated lesions, which may be associated with consecutive neurological deficits potentially influencing functional outcome. The pathogenesis of DCI is intensely debated and a multifactorial process has been proposed - inflammation, microthromboembolism, autoregulatory failure, spreading depolarization and vasospasm [2-5]. These mechanisms may contribute to DCI, 
and available data support ameliorating neuroprotective properties of $\mathrm{TH}[6,32,33]$. Clinical investigations have identified pro-inflammatory cytokines (IL-6, TNF- $\alpha$ ) to be associated with DCI and poor outcome after SAH [5, 30 ], and experimental data suggest that $\mathrm{TH}$ may reduce cytokine levels and induce upregulation of cytoprotective protein expression [33]. Causal relations of spreading depolarization with non-vasospastic DCI have been described [5] and again experimental data propose attenuation of spreading depolarization by $\mathrm{TH}$ [32]. Suggested $\mathrm{TH}$-mediated neuroprotective mechanisms, that is, decreased metabolic demand, reduced glutamate toxicity, less free-radical production, decreased neuronal calcium influx, decreased neuronal apoptosis, attenuation of blood-brain barrier and preservation of cerebral autoregulation, may increase ischemic tolerance in patients treated with hypothermia, which hypothetically may lead to fewer cerebral infarctions and potentially improved functional outcome $[6,12,14,29]$.

Limitations of this investigation: Transcranial Doppler instead of duplex examinations was performed by certified physicians; nevertheless, specificity for vasospasm detection remains debated. Reported macrovascular spasm rate may be exaggerated as low Doppler thresholds were applied and hypothermia itself may reduce cerebral blood flow limiting the interpretation of Doppler-based results but reinforce the positive associations of TH for DCI and functional outcome $[23,31]$. Within this study, DCI rate was very high as DCI detection was based exclusively on CT findings rather than on apparent neurologic deficits or multimodal imaging since assessment and executability in critically ill, mechanically ventilated SAH patients are limited. This methodology possibly overestimates DCI count and limits interpretation of causal and clinically meaningful cerebral infarctions [25]. The present study was not designed as randomized trial; low patient numbers, single center, matched controlled design and the validity of follow-up mRS assessment make it difficult to interpret the outcome findings, thus residual confounding by indication cannot be completely excluded. Importantly, this investigation has a pilot study character, which is exploratory in nature and associations of TH may be influenced by the small sample size demanding appropriately designed future trials.

\section{Conclusion}

This pilot study seems to indicate that early and prolonged TH may be beneficial for patients with severe SAH by reducing macrovascular spasm, DCI and possibly af- fecting functional outcome. Future randomized controlled studies investigating $\mathrm{TH}$ in selected SAH patients with a high DCI risk seem warranted.

\section{Acknowledgments}

We like to thank Jonathan Reichl and Henning Abel for helping with logistics and clinical data acquisition as well as Dr. Inken Martin for valuable discussions.

\section{Contributions}

J.B.K., R.K. and H.B.H. designed the study and wrote the manuscript. J.B.K., S.T.G. and D.M. evaluated clinical data. A.D. and S.P.K. obtained all neuroradiological data. J.B.K., A.P. and D.S. performed ultrasonography examinations. S.S. and I.Y.E. helped with statistical analyses and critically revised the manuscript.

\section{Disclosure Statement}

J.B.K. has received travel grants from EMSCools and D.S. from Zoll medical and Bard medical. R.K. and S.S. have received travel grants and speaker's honoraria from Zoll medical, Bard medical, Seiratherm and EMSCools. The other authors have nothing to disclose. All authors have read the manuscript, agreed with the contents and have approved the final version of the manuscript.

\section{Financial Support}

The study was partially funded by the ELAN program (12-0104-1; FSt 56610036) of the Friedrich-Alexander University Erlangen-Nuremberg.

References

1 Steiner T, Juvela S, Unterberg A, Jung C, Forsting M, Rinkel G: European stroke organization guidelines for the management of intracranial aneurysms and subarachnoid haemorrhage. Cerebrovasc Dis 2013;35:93-112.

2 Macdonald RL: Delayed neurological deterioration after subarachnoid haemorrhage. Nat Rev Neurol 2014;10:44-58.

3 Budohoski KP, Czosnyka M, Kirkpatrick PJ, Smielewski P, Steiner LA, Pickard JD: Clinical relevance of cerebral autoregulation following subarachnoid haemorrhage. Nat Rev Neurol 2013;9:152-163.

4 Woitzik J, Dreier JP, Hecht N, Fiss I, Sandow N, Major S, Winkler M, Dahlem YA, Manville J, Diepers M, Muench E, Kasuya H, Schmiedek P, Vajkoczy P: Delayed cerebral ischemia and spreading depolarization in absence of angiographic vasospasm after subarachnoid hemorrhage. J Cereb Blood Flow Metab 2012; 32:203-212. 
5 Leng LZ, Fink ME, Iadecola C: Spreading depolarization: a possible new culprit in the delayed cerebral ischemia of subarachnoid hemorrhage. Arch Neurol 2011;68:31-36.

6 Linares G, Mayer SA: Hypothermia for the treatment of ischemic and hemorrhagic stroke. Crit Care Med 2009;37(7 suppl):S243S249.

7 Mild therapeutic hypothermia to improve the neurologic outcome after cardiac arrest. N Engl J Med 2002;346:549-556.

8 Nielsen N, Wetterslev J, Friberg H: Targeted temperature management after cardiac arrest. N Engl J Med 2014;370:1360.

9 van der Worp HB, Macleod MR, Kollmar R: Therapeutic hypothermia for acute ischemic stroke: ready to start large randomized trials? J Cereb Blood Flow Metab 2010;30:10791093.

10 Kollmar R, Staykov D, Dörfler A, Schellinger PD, Schwab S, Bardutzky J: Hypothermia reduces perihemorrhagic edema after intracerebral hemorrhage. Stroke 2010;41:1684-1689.

11 Kollmar R, GebhardtB, SchwabS: [EuroHYP-1 trial: EU-funded therapy study on the effectiveness of mild therapeutic hypothermia for acute ischemic stroke]. Nervenarzt 2012;83: 1252-1259.

12 Török E, Klopotowski M, Trabold R, Thal SC, Plesnila N, Schöller K: Mild hypothermia (33 degrees $\mathrm{C}$ ) reduces intracranial hypertension and improves functional outcome after subarachnoid hemorrhage in rats. Neurosurgery 2009;65:352-359; discussion 359.

13 Todd MM, Hindman BJ, Clarke WR, Torner JC: Mild intraoperative hypothermia during surgery for intracranial aneurysm. N Engl J Med 2005;352:135-145.

14 Seule MA, Muroi C, Mink S, Yonekawa Y, Keller E: Therapeutic hypothermia in patients with aneurysmal subarachnoid hemorrhage, refractory intracranial hypertension, or cerebral vasospasm. Neurosurgery 2009;64:8692; discussion 92-93.

15 Badjatia N, Strongilis E, Gordon E, Prescutti M, Fernandez L, Fernandez A, Buitrago M, Schmidt JM, Ostapkovich ND, Mayer SA: Metabolic impact of shivering during therapeutic temperature modulation: the bedside shivering assessment acale. Stroke 2008;39: 3242-3247.
16 Geurts M, Macleod MR, Kollmar R, Kremer $\mathrm{PH}$, van der Worp HB: Therapeutic hypothermia and the risk of infection: a systematic review and meta-analysis. Crit Care Med 2013;42:231-242.

17 Connolly ES Jr, Rabinstein AA, Carhuapoma JR, Derdeyn CP, Dion J, Higashida RT, Hoh BL, Kirkness CJ, Naidech AM, Ogilvy CS, Patel AB, Thompson BG, Vespa P: Guidelines for the management of aneurysmal subarachnoid hemorrhage: a guideline for healthcare professionals from the American heart association/American stroke association. Stroke 2012;43:1711-1737.

18 Hauer EM, Stark D, Staykov D, Steigleder T, Schwab S, Bardutzky J: Early continuous hypertonic saline infusion in patients with severe cerebrovascular disease. Crit Care Med 2011;39:1766-1772.

19 Voicu S, Deye N, Malissin I, Vigué B, Brun PY, Haik W, Champion S, Megarbane B, Sideris G, Mebazaa A, Carli P, Manivet P, Baud FJ: Influence of $\alpha$-stat and $\mathrm{pH}$-stat blood gas management strategies on cerebral blood flow and oxygenation in patients treated with therapeutic hypothermia after out-of-hospital cardiac arrest: a crossover study. Crit Care Med 2014;42:1849-1861.

20 Frontera JA, Claassen J, Schmidt JM, Wartenberg KE, Temes R, Connolly ES Jr, MacDonald RL, Mayer SA: Prediction of symptomatic vasospasm after subarachnoid hemorrhage: the modified fisher scale. Neurosurgery 2006;59: 21-27; discussion 21-27.

21 Hijdra A, Brouwers PJ, Vermeulen M, van Gijn J: Grading the amount of blood on computed tomograms after subarachnoid hemorrhage. Stroke 1990;21:1156-1161.

22 Kiphuth IC, Huttner HB, Breuer L, Engelhorn T, Schwab S, Köhrmann M: Vasospasm in intracerebral hemorrhage with ventricular involvement: a prospective pilot transcranial Doppler sonography study. Cerebrovasc Dis 2011;32:420-425.

23 Lysakowski C, Walder B, Costanza MC, Tramèr MR: Transcranial Doppler versus angiography in patients with vasospasm due to a ruptured cerebral aneurysm: a systematic review. Stroke 2001;32:2292-2298.

24 Lindegaard KF, Nornes H, Bakke SJ, Sorteberg W, Nakstad P: Cerebral vasospasm diag- nosis by means of angiography and blood velocity measurements. Acta Neurochir (Wien) 1989;100:12-24.

25 de Rooij NK, Rinkel GJ, Dankbaar JW, Frijns CJ: Delayed cerebral ischemia after subarachnoid hemorrhage: a systematic review of clinical, laboratory, and radiological predictors. Stroke 2013;44:43-54.

26 Vergouwen MD, Algra A, Rinkel GJ: Endothelin receptor antagonists for aneurysmal subarachnoid hemorrhage: a systematic review and meta-analysis update. Stroke 2012; 43:2671-2676.

27 Wong GK, Poon WS: Clazosentan for patients with subarachnoid haemorrhage: lessons learned. Lancet Neurol 2011;10:871; author reply 871-872.

28 Wang ZP, Chen HS, Wang FX: Influence of plas$\mathrm{ma}$ and cerebrospinal fluid levels of endothelin-1 and NO in reducing cerebral vasospasm after subarachnoid hemorrhage during treatment with mild hypothermia, in a dog model. Cell Biochem Biophys 2011;61:137-143.

29 Muroi C, Frei K, El Beltagy M, Cesnulis E, Yonekawa Y, Keller E: Combined therapeutic hypothermia and barbiturate coma reduces interleukin-6 in the cerebrospinal fluid after aneurysmal subarachnoid hemorrhage. J Neurosurg Anesthesiol 2008;20:193-198.

30 Muroi C, Hugelshofer M, Seule M, Tastan I, Fujioka M, Mishima K, Keller E: Correlation among systemic inflammatory parameter, occurrence of delayed neurological deficits, and outcome after aneurysmal subarachnoid hemorrhage. Neurosurgery 2013;72:367-375; discussion 375

31 Seule M, Muroi C, Sikorski C, Hugelshofer M, Winkler K, Keller E: Therapeutic hypothermia reduces middle cerebral artery flow velocity in patients with severe aneurysmal subarachnoid hemorrhage. Neurocrit Care 2014; 20:255-262.

32 Takaoka S, Pearlstein RD, Warner DS: Hypothermia reduces the propensity of cortical tissue to propagate direct current depolarizations in the rat. Neurosci Lett 1996;218:25-28.

33 Kaneko T, Kibayashi K: Mild hypothermia facilitates the expression of cold-inducible RNA-binding protein and heat shock protein 70.1 in mouse brain. Brain Res 2012;1466: 128-136. 